Cad. Est. Ling., Campinas, 46(1):85-90, Jan./Jun. 2004

\title{
UNE ANALYSE DU DISCOURS DE TRANSMISSION D'INFORMATIONS PRATIQUES EN FRANÇAIS
}

\author{
RUTH DE OLIVEIRA \\ (ILPGA-Sorbonne 3 / CNRS -Osterlits)
}

\begin{abstract}
RESUMO O objetivo do presente artigo é de expor alguns resultados preliminares de uma análise do discurso de transmissão de informações práticas em francês. Os resultados propostos foram obtidos por meio do estudo de traços lingüísticos, marcas da presença do(a) informante no discurso, repertoriados em situações de trocas verbais autênticas. O objetivo específico é de proceder a uma verificação do respeito (ou do não respeito) de certas regras não ditas as quais estão relacionadas com o Princípio de cooperação do discurso (ou, com P. Grice (1975) com as Máximas da conversação).
\end{abstract}

\section{PRÉAMBULE}

Issu d'un projet de recherche plus large et encore en cours de réalisation ${ }^{1}$, cet article présente quelques résultats préliminaires d'une analyse du discours de transmission d'informations pratiques en français. Les résultats proposés ont été obtenus grâce à l'étude des traces linguistiques laissées par la présence de informateur (-trice), répertoriées dans des situations d'échanges verbaux authentiques. Le but spécifique est de présenter une vérification du respect -ou du non-respect- de certaines règles non dites qui relèvent du Principe de coopération du discours (ou, avec P. Grice (1975), des Maximes de conversation).

Une introduction présente et définit les notions théoriques, les termes et la méthodologie abordés ici. La deuxième partie est consacrée à une application pratique, c'est-à-dire à l'analyse de deux types d'énoncés à caractère informatif. Une appréciation du travail réalisé sera présentée dans la troisième partie.

${ }^{1}$ Il s'agit d'un projet de recherche intitulé «Repérage des traces linguistiques de la démarche cognitive des locuteurs/rédacteurs dans la transmission d'informations pratiques en français» que j'ai présenté au CNRS en avril 2001. 
OLIVEIRA - Une analyse du discours...

\section{INTRODUCTION}

Les études portant sur les traces d'inscription du sujet (parlant et/ ou du scripteur) dans l'énoncé relèvent du domaine de « la subjectivité dans le langage » et participent de l'articulation langage et communication. Notre démarche théorique s'appuie ainsi fondamentalement sur les travaux traditionnels et pionniers de Benveniste (1966) et de Jakobson (1963). Ces travaux ont été repris et développés plus récemment par d'autres chercheurs, notamment ceux de C. Kerbrat-Orecchioni (1999) et/ou D. Maingueneau (2000) dont nous emprunterons certains concepts pratiques.

Le type de discours traité dans cet article est celui qui relève de l'information pratique: celle dont nous sommes tributaires dans notre quotidien. De ce fait, la dénomination «information pratique» est employée au sens «utilitaire» des termes. Cela renvoie donc aussi bien au domaine administratif, touristique, commercial ou encore à une demande d'emploi, d'orientation (temps, espace) etc. Il est par ailleurs possible d'observer des subdivisions dans chacun de ces domaines. Ainsi, pour ce qui est de l'administration, il y aura les démarches pour l'obtention du permis de conduire, les inscriptions à l'université, les aides sociales etc.

Lors de la transmission orale et/ou écrite de ce type d'information, l'intervention personnelle, donc subjective, de l'informateur(-trice) rompt le caractère objectif de l'acte d'informer. Cette rupture tend généralement à complexifier le traitement de l'information par le fait que l'auditeur/lecteur demandeur est porté à distinguer le contenu subjectif de la pensée de l'informateur qui se trouve associée à l'énoncé, de son contenu proprement objectif.

Ainsi dans une information du type: «Pour procéder à son inscription l'étudiant doit impérativement se présenter aux horaires d'ouverture du bureau» l'emploi de l'adverbe impérativement peut être perçu comme une trace de l'intervention du locuteur/rédacteur dans l'énoncé. Or, dans ce contexte, la valeur sémantique du verbe devoir suffit à informer sur l'obligation de respecter les horaires prévus. De ce fait, l'intervention ne peut être considérée comme pertinente. Dans l'énoncé suivant, en revanche: «adresse électronique du bureau (à utiliser après avoir lu cette page)» l'intervention du rédacteur (à utiliser après avoir lu cette page)est manifestement objective,ne serait-ce que parce qu'elle suggère l'inutilité d'un courrier de demande de renseignements alors que ceux-ci se trouvent probablement dans le document en question.

L'analyse du discours permet, on le sait, de réintroduire le sujet parlant (ou l'énonciateur) dans la formulation des règles de correspondance.

Les énoncés analysés ici sont considérés comme des activités sociales et de ce fait, soumis à des conditions de réussite. Puisque l'échange verbal est un rituel social (Goffman, Les rites d'intération, 1974) toujours réalisé dans une perspective dialogique, il entre dans un code: ensemble d'obligations imposées par l'attente de l'autre. Il s'agit d'un processus interactif et dynamique.

Les critères de réussite du discours sont liés, on l'a souligné, à la notion de Coopération (ou de Contrat de communication). Cela signifie que «les partenaires sont censés partager un certain cadre et collaborer à la réussite de cette activité commune qu'est l'échange verbal, où chacun se reconnaît et reconnaît à l'autre certains droits et 
devoirs.» (Maingueneau, 2000: 18). Pour ce faire, ils doivent respecter les Lois du discours (ou, avec Grice (75), les Maximes conversationnelles ${ }^{2}$ ).

A ce propos, compte tenu du foisonnement de perspectives autour du sujet et de nos limitations dans le cadre d'un article, notre champ d'investigation circonscrit les lois du discours les plus récurrentes. En l'occurrence la loi de la pertinence, celles de la sincérité, de l'informativité, de l'exhaustivité et les lois de modalité.

Ainsi pour présenter une vérification du respect (ou du non-respect) de l'une ces règles (et donc du principe de Coopération), notre analyse portera sur une demande d'information (type de discours «informatif») par téléphone auprès d'une université (genre «administratif»), le numéro composé étant, avec une certitude absolue, celui du correspondant recherché. Les cas de figures proposés (2) sont extraits d'un ensemble de 22 enregistrements.

\section{ANALYSE D'UN CORPUS}

\section{1 Cas de figure}

(l'informateur) - bureau du personnel enseignant, bonjour

(le demandeur) - bonjour, j'ai une question à propos du recrutement des...

(l'informateur) - je vous écoute...

(le demandeur) - Serait-il possible de connaître le nombre de candidats pour le poste numéro...

(l'informateur) - oui, une minute s'il vous plaît... oui, alors... nous avons reçu 15 candidatures pour ce poste

\section{${ }^{2}$ Ces maximes sont les suivantes:}

Maximes de quantité

N1: Make your contribution as informative as is required (for the current purposes of the exchange).

N2: Do not make your contribution more informative than is required.

Les maximes N1 et N2 expriment des conditions sur la quantité d'information à échanger. Un comportement coopératif est basé sur un échange à la fois suffisant (N1) et minimal (N2).

Maximes de qualité

Q1: Do not say what you believe to be false.

Q2: Do not say that for which you lack adequate evidence.

Les maximes Q1 et Q2 expriment des conditions sur la qualité de l'information à échanger. Si l'on considère toujours un comportement coopératif, l'échange ne doit pas comporter d'information fausse (Q1), ou que l'on ne peut pas justifier (Q2).

Maxime de relation

R1: Be relevant.

La maxime R1 exprime la pertinence de la contribution par rapport au contexte de la conversation.

Maximes de manière

M1: Avoid obscurity of expression.

M2: Avoid ambiguity.

M3: Be brief (avoid unnecessary prolixity).

M4: Be orderly

Les maximes de manière décrivent de quelle manière la contribution doit être faite. L'expression doit être claire (M1) et non ambiguë (M2). Pour cela, il faut être bref (M3) et méthodique (M4). 
OLIVEIRA - Une analyse du discours...

(le demandeur) - merci beaucoup. au revoir;

(l'informateur) - au revoir.

A l'analyse de ce premier cas de figure (1), le contrat de communication semble bien avoir été respecté. L'identification immédiate du correspondant «bureau du personnel enseignant» est un fait pertinent puisque la loi de la pertinence stipule qu'une énonciation doit être appropriée au contexte dans lequel elle intervient (Maingueneau, 2000: 20). Cet engagement de l'énonciateur dans l'acte du discours est renforcée par le «je vous écoute» et assure du coup la loi de la sincérité. Ce «je» peut par ailleurs être considéré comme un «je» distancié censé convenir à la routine énonciative de ce type cadre. Son efficacité est assurée par le fait qu'il énonce une information et qu'il l'enveloppe en quelque sorte sans pour autant être explicité dans l'énoncé. Les propos du sujet renvoient ainsi à l'institution «administration» puisqu'il est question du «bureau du personnel enseignant» et d'un «nous avons reçu...etc».

L'informateur est économe ${ }^{3}$, son discours se construit autour des phrases courtes (S/ V/P), il énonce la formulation la plus directe et infère donc des lois de modalité (: être clair). La suite de son discours se conforme d'une part, à la loi d'informativité laquelle porte sur le contenu des énoncés et doit apporter des informations nouvelles, c'est-à-dire «15 candidats pour le poste» et d'autre part, à la loi d'exhaustivité dont la finalité est de donner l'information maximale. En l'occurrence on apprend qu'il s'agit de «15 candidats» (et non pas, par exemple, d'«une quinzaine» ou encore d' «une bonne quinzaine» ${ }^{4}$ ). Passons à présent au cas de figure (2).

\subsection{Cas de figure 2 .}

(l'informateur) - allô?

(le demandeur) - bonjour, bureau du personnel enseignement de..?

(l'informateur) - ça dépend...

${ }^{3}$ Les principes d'économie sont les suivants:

E1: The expression must not convey more meaning than necessary.

E2: The expression must be the shortest possible.

E3: The more frequent the expression the shorter it must be.

Pour qu'une contribution soit la plus économique possible, il faut qu'elle comporte l'information minimale requise (E1), qu'elle soit la plus courte possible (E2) et plus une expression est fréquente, plus elle doit être courte (E3).

${ }^{4}$ La réponse par nombre approximatif est apparue dans 10 des 22 enregistrements.

${ }^{5}$ Les principes de clarté sont les suivants

C1: The expression must convey to the listener all the meanings intended by the speaker.

C3: The expression must contain redundancy.

C4: The greater the possibility for semantic confusion the greater must be the difference in expression.

Pour qu'une contribution soit la plus claire possible, elle doit contenir toute l'information que le locuteur a l'intention de communiquer (C1), éventuellement contenir des redondances (C2) et lever les ambiguïtés lorsque cela est nécessaire (C3). 
(le demandeur) - c'est à propos du recrutement des ...

(l'informateur) - c'est trop tard!

(le demandeur) - attendez, je sais bien que le recrutement est fini et ma question porte sur un autre aspect du concours...je voudrais connaître le nombre de candidats postulant pour le poste numéro...

(l'informateur) - ah... alors là franchement je n'en sais rien... de toute façon je suis très occupée il faudra rappeler...

(le demandeur) - au revoir

(l'informateur) - au revoir

Ici, contrairement au cas de figure (1) le «allô» inaugural du discours de l'informateur suivi de «ça dépend» sont vraisemblablement inappropriés au contexte (au cadre) dans lequel ils interviennent. Mais comme toute énonciation implique sa pertinence, le demandeur cherche à confirmer la pertinence de cet «allô» (s'est-il trompé de numéro?) par un «bureau du personnel enseignant?» et obtient un «ça dépend» qui souligne l'absence d'engagement de l'énonciateur et brise du coup la loi de la sincérité Or en énonçant «ça dépend» l'informateur affirme en quelque sorte qu'il s'agit bien du bureau en question. Le demandeur s'empresse alors d'apporter une précision qui renvoie - du point de vue de ce qu'il recherche - à une information maximale «c'est à propos du concours». Mais l'information qu'il obtient n'est toujours pas pertinente puisque si la réponse «c'est troptard » pourrait éventuellement répondre à une question (par ex.: ai-je encore le temps de m'inscrire au concours?) elle prend ici une valeur de commentaire. Une fois la demande d'information enfin formulée, l'informateur transgresse à nouveau les lois du discours. Ainsi, la loi de l'informativité n'est pas respectée or si le sujet parlant affirme «j'en sais rien» le «de toute façon, je suis très occupée» laisse supposer qu'il peut (pourrait) éventuellement disposer de l'information sollicitée. Cette absence d'engagement de sa part représente une rupture vis-à-vis des principes de clarté et renforce le non respect de la loi de la sincérité. L'emploi de «franchement»-adverbe employé quelquefois dans un but stratégique (lorsque, par exemple, l'un des interlocuteurs veut préserver sa face positive, sa façade sociale, l'image valorisante de soi qu'on s'efforce de présenter à l'extérieur)nous montre alors qu'en essayant de faire valoir de façon arbitraire la loi de la sincérité le locuteur risque d'entrer en conflit avec d'autres lois relevant de la politesse. Au lieu de se contenter du cadre générique habituel dans ce type d'échange, l'informateur matérialise en quelque sorte l'anonymat et se met en relation directe avec les autres (dans ce cas, avec le demandeur en question).

\section{CONCLUSION}

L'objectif de cet article était de présenter quelques résultats préliminaires concernant une analyse effectuée sur le discours de transmission d'informations pratiques. Nous avons pu ainsi observer que les traces laissées par la présence de l'énonciateur dans son (ce type de) discours constituent une source importante d'informations, susceptibles de nous guider vers une esquisse de profil de l'informateur (-trice) à travers l'usage qu'il fait de la langue. 
OLIVEIRA - Une analyse du discours...

Puisque la communication verbale est aussi une relation sociale, il va de soi qu'elle est soumise aux règles de ce qu'on appelle la politesse. Par conséquent, transgresser une (ou les) lois du discours (parler à côté du sujet, être obscur, ne pas donner les informations requises, etc) (Maingueneau, 2000: 23) c'est s'exposer à être catalogué d'impoli. Par ailleurs, pour ce qui est de l'impact du comportement langagier de l'informateur (-trice) sur l'auditeur/lecteur, même si cela appartient à d'autres compétences, ces résultats partiels pourraient néanmoins offrir des pistes fécondes.

\section{RÉFÉRENCES BIBLIOGRAPHIQUES}

GOFFMAN, E. (1979). Les rites d'interaction. Paris: Ed. de Minuit.

GRICE, P. (1979). Logique et conversation, in Communication, n. 30.

KERBRAT-ORECCHIONI, C. (1999). L'énonciation: De la subjectivité dans le langage. Paris: Armand Colin.

MAINGUENEAU, D. (2000). Analyser les textes de communication. Paris: Nathan. 